\title{
Risco do desenvolvimento de doenças renais crônicas versus perfil nutricional de
} pacientes hospitalizados

\author{
Risk of the development of chronic kidney diseases versus nutritional profile of hospitalized \\ patients \\ Riesgo de desarrollo de enfermidades renales crónicas versus perfil nutricional de pacientes \\ hospitalizados
}

\author{
Moisés Iasley Lima Vasconcelos \\ ORCID: https://orcid.org/0000-0002-8724-1063 \\ Centro Universitário Estácio do Ceará, Brasil \\ E-mail: moisesasley@gmail.com \\ Arnaldo Teixeira Braga Júnior \\ ORCID: https://orcid.org/0000-0003-1689-3280 \\ Centro Universitário Estácio do Ceará, Brasil \\ E-mail: e14.arnaldojunior@gmail.com \\ Mariana Bezerra Gomes \\ ORCID: https://orcid.org/0000-0003-3832-7511 \\ Centro Universitário Estácio do Ceará, Brasil \\ E-mail: marigomesmb1998@gmail.com \\ Gerusa Matias dos Santos \\ ORCID: https://orcid.org/0000-0002-6261-8565 \\ Centro Universitário Estácio do Ceará, Brasil \\ E-mail: gerusa.santos@estacio.br \\ André Ricardo Bindá de Borba \\ ORCID: https://orcid.org/0000-0002-7147-4145 \\ Centro Universitário Estácio do Ceará, Brasil \\ E-mail: andrebinda@hotmail.com \\ Carolina Drummond Barboza \\ ORCID: https://orcid.org/0000-0002-7392-2562 \\ E-mail: carolina.cdb@isgh.org.br \\ Universidade Estadual do Ceará, Brasil \\ Antônio Augusto Ferreira Carioca \\ ORCID: https://orcid.org/0000-0002-1194-562X \\ Universidade de Fortaleza, Brasil \\ E-mail: Carioca@unifor.br \\ Tatiana Uchôa Passos \\ ORCID: https://orcid.org/0000-0002-7604-5948 \\ Centro Universitário Estácio do Ceará, Brasil \\ E-mail: tatiana.passos@estacio.br
}

\begin{abstract}
Resumo
Correlacionar o estado nutricional com o risco de desenvolvimento da doença renal crônica. A coleta de dados foi realizada em um hospital de Fortaleza-CE. Sendo realizada a avaliação nutricional em adultos e idosos com aplicação de dois questionários, denominados: Screening For Occult Renal Disease (SCORED) e Questionário de Frequência Alimentar (QFA), juntamente com exames bioquímicos e ingestão hídrica. Vale ressaltar que os dados coletados foram tabulados no programa Microsoft Excel versão 2016, realizados testes estatísticos para confiabilidade das correlações em busca do cruzamento entre perfil nutricional e o desenvolvimento de DRC no paciente hospitalizado. A amostra foi composta por 260 voluntários, sendo 58,1\% do sexo masculino, sendo o maior percentual de $34,6 \%$ (90) com idades entre 40-69, onde 40\% (104) da amostra é negra e renda per capita mediana inferior a um saláriomínimo. Quanto ao Índice de Massa Corporal (IMC) 15,8\% (41) dos pacientes adultos de ambos os sexos são eutróficos, já o percentual encontrado para pacientes idosos foi de 20,4\% (53) classificados assim, em magreza. Conforme constatou o questionário SCORED 75,4\% dos avaliados possui o risco de desenvolver DRC. Quanto aos biomarcadores, os valores de ureia apresentaram-se inadequados em 62,7\% dos avaliados e 42,7\% estavam com TFG em estágio 2. É de suma relevância a monitorização e reavaliação frequente dos pacientes por parte dos profissionais. $\mathrm{O}$ acompanhamento nutricional visa promover uma melhor qualidade de vida, minimizando os riscos nutricionais em âmbito hospitalar.
\end{abstract}


Palavras-chave: Doença Renal Crônica; Perfil nutricional; Rastreamento. Abstract

Correlate nutritional status with the risk of developing chronic kidney disease. Data collection was performed at a hospital in Fortaleza-CE. Nutritional assessment was carried out in adults and elderly with the application of two questionnaires, called: Screening For Occult Renal Disease (SCORED) and Food Frequency Questionnaire (FFQ), along with biochemical tests and water intake. It is noteworthy that the collected data were tabulated in Microsoft Excel version 2016, statistical tests were performed for the reliability of the correlations in search of a cross between nutritional profile and the development of CKD in hospitalized patients. The sample consisted of 260 volunteers, $58.1 \%$ male, with the highest percentage being 34.6\% (90) aged between 40-69, where 40\% (104) of the sample is black and with per capita income median less than a minimum wage. As for the Body Mass Index (BMI), 15.8\% (41) of adult patients of both sexes are eutrophic, whereas the percentage found for elderly patients was 20.4\% (53) classified as thin. As verified by the SCORED questionnaire, 75.4\% of the evaluated have the risk of developing CKD. As for biomarkers, urea values were inadequate in $62.7 \%$ of those evaluated and $42.7 \%$ had stage 2 GFR. It is extremely important to monitor and frequently reassess patients by professionals. Nutritional monitoring aims to promote a better quality of life, minimizing nutritional risks in the hospital environment.

Keywords: Chronic Kidney Disease; Nutritional profile; Tracking.

\section{Resumen}

Correlacionar el estado nutricional con el riesgo de desarrollar enfermedad renal crónica. La recolección de datos se realizó en un hospital de Fortaleza-CE. La evaluación nutricional se realizó en adultos y ancianos con la aplicación de dos cuestionarios, denominados: Screening For Occult Renal Disease (SCORED) y Food Frequency Questionnaire (FFQ), junto con pruebas bioquímicas e ingesta de agua. Es de destacar que los datos recolectados fueron tabulados en Microsoft Excel versión 2016, se realizaron pruebas estadísticas para la confiabilidad de las correlaciones en busca de un cruce entre el perfil nutricional y el desarrollo de ERC en pacientes hospitalizados. La muestra estuvo conformada por 260 voluntarios, $58.1 \%$ hombres, siendo el porcentaje más alto 34.6\% (90) con edades entre 40-69, donde el 40\% (104) de la muestra es de raza negra y con una mediana de ingreso per cápita menor al salario mínimo. En cuanto al Índice de Masa Corporal (IMC), el 15,8\% (41) de los pacientes adultos de ambos sexos son eutróficos, mientras que el porcentaje encontrado para los ancianos fue del 20,4\% (53) clasificados como delgados. Como verifica el cuestionario SCORED, el 75,4\% de los evaluados tienen riesgo de desarrollar ERC. En cuanto a los biomarcadores, los valores de urea fueron inadecuados en el $62,7 \%$ de los evaluados y el $42,7 \%$ presentaba TFG en estadio 2. Es de suma importancia el seguimiento y reevaluación frecuente de los pacientes por parte de los profesionales. El seguimiento nutricional tiene como objetivo promover una mejor calidad de vida, minimizando los riesgos nutricionales en el entorno hospitalario.

Palabras clave: Enfermedad renal crónica; Perfil nutricional; Seguimiento.

\section{Introdução}

A Doença Renal Crônica (DRC) caracteriza-se por meio de uma lesão renal, com perda gradativa, progressiva e irreversível de todas as funções: glomerular, endócrina e tubular, independente dos fatores etiológicos envolvidos nessa lesão. Possui progressão lenta podendo se estender a meses ou anos, nas fases iniciais costuma ser assintomático o que torna seu diagnóstico tardio, sendo de alta prevalência em pacientes hospitalizados (Fiaccadori et al., 2021).

Os rins têm múltiplas funções como: excreção, filtração dos processos metabólicos, produção de hormônios, controle do equilíbrio hidroeletrolítico, metabolismo ácido-básico e da pressão arterial. Do ponto de vista clínico, a função excretora é aquela que tem maior correlação com os desfechos clínicos, logo, todas as funções renais costumam declinar de forma paralela com a sua função excretora (Brasil, 2014).

Independente da etiologia da doença de base, os principais desfechos em pacientes com DRC são as suas complicações: (anemia, acidose metabólica, desnutrição e alteração do metabolismo de cálcio e fósforo) decorrentes da perda funcional dos rins, o óbito (principalmente por causas cardiovasculares) é muito comum nos pacientes com DRC e a necessidade de terapia renal substitutiva minimiza as complicações associado ao quadro clínico do paciente (Kirsztajn et al., 2011).

Essa patologia pode interferir no metabolismo, absorção, biodisponibilidade e excreção de nutrientes como: potássio, fósforo, cálcio, zinco, vitamina D entre outros, podendo ocasionando distúrbios nutricionais, resultando em desequilíbrio renal 
e metabólico que afetam diretamente e indiretamente o estado nutricional dos portadores de DRC (Cozzolino \& Cuppari; 2019).

Representantes da Sociedade Brasileira de Nefrologia (SBN), relatam que a doença renal crônica (DRC) já pode ser considerada epidêmica, visto que atinge um a cada dez adultos, e a incidência está aumentando. Hoje no Brasil, 133 mil pessoas dependem de diálise, número que cresceu $100 \%$ nos últimos dez anos. Anualmente, mais de 20 mil pacientes entram em hemodiálise, com taxa de mortalidade de $15 \%$ ao ano. A prevenção é a base da campanha em 2020, mas a terapia renal está em crise. Somente $7 \%$ das cidades dispõe do serviço de diálise. Atualmente as clínicas credenciadas enfrentam subfinanciamento e perdem capacidade de atendimento e de investimento em qualidade. O resultado se reflete na superlotação e na redução de vagas para novos pacientes (Brasil, 2020).

A prevenção da DR gera menos gastos para o sistema de saúde comparado aos valores que seria usado com tratamento. Dados da Sociedade Brasileira de Nefrologia (SBN), informam que nos últimos cinco anos, pessoas submetidas a diálise teve um aumento de 4,2 vezes mais do que o número de centro de diálises em funcionamento no país, sendo que mais de 80\% destes é custeado pelo Sistema Único de Saúde (SUS) (Thomé et al., 2019).

A avaliação nutricional é fundamental nos cuidados dos pacientes hospitalizados, pois fornece o diagnóstico do estado nutricional do paciente que interfere diretamente no seu desenvolvimento clínico, nesse contexto, o nutricionista está inserido juntamente com uma equipe de profissionais planejando protocolos e programas de segurança do paciente, permitindo a criação de um plano de cuidado integral. A desnutrição é um fator negativo para o desenvolvimento da DRC, pois está relacionada a um número maior de complicações hemodinâmicas, além da maior frequência e duração das internações hospitalares, em sua maioria, a desnutrição é associada à ingestão alimentar insuficiente, devido às terapias substitutivas, restrições alimentares, e/ou, principalmente, ao hipercatabolismo do paciente (Vasconcelos et al., 2021).

Em resposta à vida moderna, a falta de tempo e o apelo à praticidade, a indústria consegue convencer a população com a proposta de produtos prontos para consumo ou de preparação rápida e fácil, de baixo custo, tornando-os aceitáveis ao paladar às custas da adição de grandes quantidades de óleo, açúcar e sal, conservantes, purinas e açúcares se o consumo for elevado estar ocasionando o aumento de peso e o surgimento de DRC (Vasconcelos et al., 2021).

Nesse contexto, pacientes hospitalizados portadores de DRC estão predispostos à desnutrição é possível avaliar e identificar a ingestão alimentar detalhada do paciente, com os métodos como o Questionário De Frequência Alimentar (QFA), no qual é possível verificar as alterações nas quantidades e qualidades dos alimentos consumidos pelo indivíduo (Krause et al., 2018).

A terapia nutricional no paciente com DRC desde que planejada, individualizada e de boa aceitação, possibilita a manutenção e o restabelecimento do estado nutricional, portanto, a avaliação nutricional de pacientes hospitalizados, permite o diagnóstico do estado nutricional e contribui para a recuperação e/ou manutenção destes, proporcionando uma intervenção nutricional durante sua internação (Bousquet-Santos, 2019).

O rastreamento da DRC se dá por um conjunto de fatores associados, que indicam alerta para uma predisposição da patologia, segundo o Instituto Nacional de Saúde norte americano, biomarcadores são explicados como indicadores quantitativos de processos biológicos ou patológicos utilizados para fins de diagnóstico ou de monitorização da terapêutica (Cecconello, 2019).

Os meios utilizados para identificar e diagnosticar o paciente com DRC são: Taxa de Filtração Glomerular (TFG), o exame sumário de urina (EAS) e um exame de imagem, preferencialmente a ultrassonografia dos rins e vias urinárias sendo relevantes assim para o diagnóstico. O cálculo da TFG é recomendado para os pacientes que apresentem riscos de desenvolver DRC, dosando sua creatinina sérica e avaliando os valores obtidos (Brasil, 2014). 
Portanto, o presente estudo teve como objetivo associar o estado nutricional com o risco de desenvolvimento da doença renal crônica em pacientes hospitalizados.

\section{Metodologia}

O presente estudo trata-se de um estudo transversal, quantitativo, descritivo e analítico. A pesquisa foi realizada nos meses de agosto a novembro de 2021, em um hospital público de Fortaleza-CE. A instituição possui atendimento terciário, onde, o público engloba pacientes transferidos de outras unidades hospitalares que necessitam de um tratamento especializado, com prevalência das seguintes patologias: Diabetes Mellitus, Hipertensão Arterial, Doenças Renais, Doenças Hepáticas e Pancreáticas, a coleta foi realizada nas seguintes enfermarias: em quatro clínicas médicas e clínica AVC subaguda.

O público-alvo para coleta de dados foram os pacientes com idade igual ou superior a 20 anos de ambos os sexos e não portadores de DRC foram excluídos na coleta de dados do presente estudo gestantes, pacientes em uso de terapia nutricional enteral e parenteral, pacientes com transtornos mentais e em cuidados paliativos. Para o cálculo amostral levou-se em consideração o nível de confiança de 95\%, para margem de erro 5\%, considerando uma média de 175 leitos, resultando em uma amostra de 121 entrevistados, logo, o atual estudo teve um quantitativo amostral de 260 voluntários.

A primeira etapa para o levantamento de dados foi realizada por meio de registro de informações socioeconômicas e antropométricas, a partir de entrevista direta com os pacientes e análise de prontuário e seus respectivos valores antropométricos e exames bioquímicos. A coleta de dados teve início com a aplicação de um questionário, registrado pelos próprios pesquisadores contendo informações sobre: nome, data de nascimento, idade, sexo, raça, hereditariedade, dados antropométricos e realidade financeira, após o preenchimento da ficha de identificação foram traçadas as características do público analisado.

Os dados antropométricos levantados foram direcionados à avaliação do estado nutricional, tendo como referência o índice de massa corpórea (IMC) de acordo com a (OMS, 1995), para a realização desse método foram aferidos peso e altura registrados na admissão do paciente com capacidade funcional satisfatória, para os pacientes acamados, hemodinamicamente instáveis foram estimados peso e altura de acordo com (Chumlea et al., 1985), sendo aferidos a estatura do joelho e a circunferência do braço. Para classificar os voluntários foi utilizado como referência a (OMS, 1995 \& 1998) para voluntários adultos (idade > 20 anos), no caso dos voluntários idosos usou-se (idade > 60 anos), classificados pelo IMC de acordo com o autor (Lipschitz, 1994/ Ministério da Saúde - Brasil 2006), para classificar o estado nutricional dos voluntários de acordo com a circunferência do braço (CB) em adultos e idosos e a circunferência da panturrilha (CP), sendo (OMS, 1995, Coelho Et Al., Chumlea et al., 1995), para CP e (Frisancho, 1990) para CB.

Para o inquérito alimentar foi realizada uma investigação através de um questionário de frequência alimentar (QFA), o mesmo com o objetivo de verificar a ingestão de alimentos com o alto teor de proteína e sódio, que estão associados com desenvolvimento da doença renal crônica, em sinergia com outros hábitos como: ingestão hídrica deficiente e o consumo exacerbado de álcool. O QFA utilizado é uma versão adaptada do inquérito de consumo alimentar ISACAMP-NUTRI 2014, foi feita a exclusão de grupos alimentares que não estão diretamente relacionados com o desenvolvimento de doença renal, mantendo como base alimentos proteicos e com alto teor de sódio.

Para o rastreamento da DRC, foi aplicada uma versão adaptada do questionário Screening For Occult Renal Disease SCORED, que tem como objetivo rastrear a doença renal crônica em grupos populacionais. Prezando pela maior fidedignidade dos resultados, optou-se por preencher o questionário baseado nas informações constantes em prontuário, haja vista a dificuldade de compreensão e letramento de alguns pacientes e seus acompanhantes. Além disso, foram incluídos mais alguns itens relativos a parâmetros bioquímicos (TFG, Creatina e Ureia), considerando a relevância destes para o diagnóstico e acompanhamento da DRC. 
Além dos exames bioquímicos citados na ferramenta de rastreio, inseriu-se a verificação da adequação dos seguintes minerais: cálcio, magnésio, fósforo, potássio e sódio, para associar com a progressão da doença, os dados foram retirados diretamente do prontuário hospitalar do paciente. Para os valores referência de adequação dos parâmetros bioquímicos citados, foram utilizadas as referências disponíveis pelo laboratório de análises clínicas PREVILAB, sendo ele o laboratório utilizado nas unidades de saúde vinculadas ao Instituto de Saúde e Gestão Hospitalar (ISGH).

Para avaliar a ingestão hídrica utilizamos um miniquestionário que contém informações da ingestão de água do paciente, podendo variar a ingestão < 1L ou > 3L diário, tendo em vista que a ingestão insuficiente está associada com o aparecimento da DRC. Para a interpretação de dados, foram tabulados no programa Microsoft Excel versão 2016. Onde são realizados testes estatísticos de confiabilidade das correlações, dentre eles, em busca do cruzamento entre estado nutricional e o desenvolvimento de DRC no paciente hospitalizado. Esta pesquisa atendeu aos aspectos éticos em cumprimento da resolução de no 466/2012 aprovada pelo Conselho Nacional de Saúde (CNS).

\section{Resultados e Discussão}

A pesquisa obteve um valor amostral de 260 voluntários, de ambos os sexos, os resultados são expressos em Tabelas nas quais pode-se perceber características sociodemográficas avaliadas em pacientes hospitalizados que a prevalência de idade foi de 60-79 anos (34,6\% da amostra) e com menor destaque foram os que apresentavam idade >80 anos (13,4\%), em relação a variante raça, os negros tiveram maior participação (40\% da amostra).

Tabela 1: Características sociodemográficas avaliadas em pacientes hospitalizados $(\mathrm{n}=260)$ e caracterização do risco para Doença Renal Crônica (DRC). Fortaleza, 2021.

\begin{tabular}{|c|c|c|}
\hline Variáveis Sociodemográficas Avaliadas em Pacientes Hospitalizados & $\mathbf{n}$ & $\%$ \\
\hline \multicolumn{3}{|l|}{ Idade: } \\
\hline 20-39 anos & 48 & 18,5 \\
\hline $40-59$ anos & 87 & 33,5 \\
\hline $60-79$ anos & 90 & 34,6 \\
\hline$>80$ anos & 35 & 13,4 \\
\hline \multicolumn{3}{|l|}{ Sexo: } \\
\hline Masculino: & 151 & 58,1 \\
\hline Feminino: & 109 & 41,9 \\
\hline \multicolumn{3}{|l|}{ Raça: } \\
\hline Negra & 104 & 40,0 \\
\hline Branca & 92 & 35,4 \\
\hline Outras & 64 & 24,6 \\
\hline \multicolumn{3}{|l|}{ Renda Familiar: } \\
\hline$<1$ Salário & 116 & 44,6 \\
\hline >1 Salário & 85 & 32,7 \\
\hline 1 - 2 Salários & 50 & 19,2 \\
\hline$>2$ Salários & 9 & 3,5 \\
\hline \multicolumn{3}{|l|}{ Algum caso de DRC na família? } \\
\hline Sim & 167 & 64,2 \\
\hline Não & 93 & 35,8 \\
\hline \multicolumn{3}{|l|}{ Risco para o desenvolvimento da DRC: } \\
\hline $\begin{array}{l}\text { Se você marcou (4) ou mais pontos: Você tem } 1 \text { chance em } 5 \text { de ter } \\
\text { a doença renal crônica }\end{array}$ & $196 *$ & $75,4^{*}$ \\
\hline $\begin{array}{l}\text { Se você marcou ( } 0-3) \text { pontos: Você, provavelmente, não tem risco de desenvolver a doença renal } \\
\text { crônica agora. }\end{array}$ & 64 & 24,6 \\
\hline
\end{tabular}

Fonte: Dados da pesquisa. 
Com base nas respostas obtidas e representadas na Tabela 1, pode-se perceber que a prevalência de idade foi de 60-79 $\operatorname{anos}(34,6 \%$ da amostra) e com menor destaque foram os que apresentavam idade >80 anos (13,4\%), em relação a variante raça, os negros tiveram maior participação (40\% da amostra) e os brancos somaram (35,3\%), sobre a condição financeira dos entrevistados a maioria $(44,6 \%)$ possuía menos de um salário mínimo e minoria $(3,5 \%)>2$ salários. Quando perguntado aos entrevistados sobre casos de Doença Renal Crônica na família 167 (64,2 \%) responderam que já houve casos. Mediante as perguntas do Screening For Occult Renal Disease - SCORED resultou que de 260 voluntários, 196 (75,3\%) possuem chances de desenvolver Doença Renal Crônica, em contrapartida apenas 64 entrevistados (24,6\%) não possui risco de desenvolver DRC no momento.

Em um estudo realizado em Belém-PA no ano de 2018 com a população idosa, dividiu os resultados em graus de riscos para desenvolver DRC, foi classificado respectivamente em risco leve, moderado e alto, no qual o risco leve corresponde $\leq$ ao grau III, já o risco moderado é $>$ que grau III e $<$ que grau VII, e por último o risco alto que vai do intervalo $\geq$ ao grau VII á $\leq$ grau X, Desta maneira, pode-se inferir que cerca de $75 \%$ dos participantes encontram-se com risco moderado para DRC (Alfaia et al., 2018). Valor significativo de análise semelhante à pesquisa supracitada. O impacto do diagnóstico precoce da DRC é de suma relevância, pois possibilita a diminuição da mortalidade de causa cardiovascular; a redução do custo do tratamento, por retardar a progressão da DRC e por postergar a implantação da Terapia Renal Substitutiva (TRS); a melhora da qualidade de vida dos pacientes; o encaminhamento no tempo certo para o nefrologista (Trivedi, 2010; Bastos \& Kirsztajn 2011; Trivedi et al., 2012).

Uma pesquisa publicada em 2016 realizada no Ceará aponta que a ferramenta SCORED foi capaz de apontar risco de DRC nos indivíduos idosos, de classe social mais baixa, porém, na análise multivariada, classe social, sexo, idade não sendo variáveis preditoras independentes, mas sim associadas. A capacidade do SCORED (sem depender de exames laboratoriais) em predizer nível mais elevado de creatinina e TFG mais rebaixada entre os sujeitos da amostra (Santos \& Neto, 2016).

Tabela 2: Perfil antropométrico, segundo Índice de Massa Corporal (IMC), Circunferência do braço (CB) e circunferência da panturrilha (CP) de pacientes hospitalizados. Fortaleza, 2021. (n: 260).

\begin{tabular}{|c|c|c|c|c|c|}
\hline CLASSIFICAÇÃO DO IMC & n & $\%$ & CIRCUNFERÊNCIAS & $\mathbf{n}$ & $\%$ \\
\hline IMC - Adulto: & 135 & 51,92 & CB: & 260 & 100 \\
\hline Magreza $\mathrm{G}^{0} 3$ & 9 & 3,5 & & & \\
\hline Magreza $G^{0} 2$ & 4 & 1,5 & $<70 \%$ - Desnutrição grave & 20 & 7,7 \\
\hline Magreza $G^{0} 1$ & 9 & 3,5 & 70-80\% - Desnutrição moderada & 36 & 13,9 \\
\hline Eutrofia & 41 & 15,8 & 80-90\% - Desnutrição leve & 59 & 22,7 \\
\hline Sobrepeso & 36 & 13,8 & $90-110 \%$ - Eutrofia & 63 & 24,2 \\
\hline Obesidade $G^{0} 1$ & 25 & 9,6 & $110-120 \%$ - Sobrepeso & 46 & 17,7 \\
\hline Obesidade $\mathrm{G}^{0} 2$ & 6 & 2,3 & $>120 \%$ - Obesidade & 36 & 13,8 \\
\hline Obesidade $\mathrm{G}^{0} 3$ & 5 & 1,9 & & & \\
\hline IMC - Idoso & 125 & 48,1 & CP: & 125 & 100 \\
\hline Magreza & 53 & 20,4 & & & \\
\hline Eutrofia & 42 & 16,2 & $<31 \mathrm{~cm}$ - Desnutrição & 79 & 63,2 \\
\hline Sobrepeso & 30 & 11,5 & $>31 \mathrm{~cm}$ - Eutrofia & 46 & 36,8 \\
\hline
\end{tabular}

CP: Circunferência da panturrilha - CB: Circunferência do braço. Fonte: Dados da pesquisa.

Na Tabela 2 é possível observar que 15,8\% (41) dos pacientes adultos de ambos os sexos se encontram em eutrofia, já para os índices de desnutrição, pode-se observar que 3,5\% (9) dos pacientes encontram-se classificados em magreza grau I e 
3,5\% (9) em magreza grau III. Em pacientes idosos, o percentual encontrado foi: 16,2\% (42) encontram-se em eutrofia e já $20,4 \%$ (53) podem ser classificados em magreza.

O IMC é um método para diagnosticar o estado nutricional de pacientes, em um estudo realizado por Santos et al. (2013), segundo a classificação pela OMS, nenhum paciente apresentava quadro de desnutrição, $56,7 \%(\mathrm{n}=17)$ estavam eutróficos e 43,3\% (n=13) estavam acima do peso.

Em outro estudo realizado em 2018 com pacientes idosos internados em um hospital municipal na cidade de Caxias/MA (Silva et al., 2019), podemos observar que 40,0\% da população analisada estava com o peso adequado para a altura segundo o IMC, no entanto, observa-se o elevado percentual de idosos com desnutrição $(33,3 \%)$ e obesidade $(26,7 \%)$. Idosos desnutridos apresentam índices de morbidade e mortalidade maiores, e estão mais suscetíveis a complicações durante o período de internação e assim permanecem no hospital por mais tempo e requerem maior cuidado médico (Silva et al., 2019). A desnutrição em indivíduos hospitalizados correlaciona-se com o crescimento da frequência de intercorrências clínicas e mortalidade, sendo também associada a um maior período de internamento hospitalar, maior risco de infecções e impacto negativo nos custos para o serviço de saúde. A desnutrição em pessoas hospitalizadas decorre de uma gama de condições, prévias e posteriores à hospitalização, podendo ser atribuída à doença e/ou à terapia aplicada (Aquino \& Philippi, 2011; Campelo et al., 2007).

Na Tabela 2, quanto aos indicadores de massa muscular, pode-se observar que em relação a Adequação da CB, 24,2\% (63) encontram-se em eutrofia, 22,7\% (59) encontram-se em desnutrição leve, 13,9\% (36) classificam-se em desnutrição moderada, 7,7\% (20) encontram-se em desnutrição grave, 17,7 (46) classificam-se em sobrepeso e 13,8\% (36) encontram-se em obesidade segundo Blackburn e Thornton (1979). Em relação à classificação da CP, 63,20\% (79) encontramse em desnutrição e 36,80\% (46) encontram-se eutróficos segundo os parâmetros da OMS (1995).

No estudo realizado por Rosa a respeito do consumo alimentar e estado nutricional de 48 idosos residentes em uma instituição filantrópica, observou-se que a maioria dos idosos, de ambos os sexos, estava com excesso de peso segundo a classificação do IMC. A adequação da CB indicou maior prevalência de desnutrição e a partir da avaliação da CP apenas as mulheres apresentavam depleção de massa muscular (Rosa et al., 2020).

Tabela 3: Teste de qui-quadrado após análise da correlação do estado nutricional de adultos com risco do desenvolvimento de doença renal crônica através da aplicação do questionário SCORED. Fortaleza, 2021.

\begin{tabular}{|c|c|c|c|c|c|c|}
\hline \multirow[b]{2}{*}{ IMC (adultos) } & & \multicolumn{2}{|c|}{$\begin{array}{l}\text { Classificação de risco para doença } \\
\text { renal crônica }\end{array}$} & \multirow[b]{2}{*}{ Total } & \multirow[b]{2}{*}{$\mathbf{p}$} & \multirow[b]{2}{*}{$\mathbf{X}^{2}$} \\
\hline & & Risco & Baixo risco & & & \\
\hline \multirow[t]{2}{*}{ Magreza } & Observados & 9 & 12 & 21 & \multirow{10}{*}{$\mathbf{0 , 4 2 7 3}$} & \multirow{10}{*}{2,78} \\
\hline & Esperados & 12,20 & 8,80 & 21,00 & & \\
\hline \multirow[t]{2}{*}{ Eutrofia } & Observados & 26 & 16 & 42 & & \\
\hline & Esperados & 24,40 & 17,60 & 42,00 & & \\
\hline \multirow[t]{2}{*}{ Sobrepeso } & Observados & 23 & 13 & 36 & & \\
\hline & Esperados & 20,91 & 15,09 & 36,00 & & \\
\hline \multirow[t]{2}{*}{ Obesidade } & Observados & 21 & 16 & 37 & & \\
\hline & Esperados & 21,49 & 15,51 & 37,00 & & \\
\hline \multirow[t]{2}{*}{ Total } & Observados & 79 & 57 & 136 & & \\
\hline & Esperados & 79,00 & 57,00 & 136,00 & & \\
\hline
\end{tabular}

Fonte: Dados da pesquisa. 
Tabela 4: Teste de qui-quadrado da razão de verossimilhança, após análise da correlação do estado nutricional de idosos com risco do desenvolvimento de doença renal crônica através da aplicação do questionário SCORED. Fortaleza, 2021.

\begin{tabular}{|c|c|c|c|c|c|c|}
\hline \multirow[b]{2}{*}{ IMC (idoso) } & & \multicolumn{2}{|c|}{$\begin{array}{c}\text { Classificação de risco para doença renal } \\
\text { crônica }\end{array}$} & \multirow[b]{2}{*}{ Total } & \multirow[b]{2}{*}{ p } & \multirow[b]{2}{*}{ RV } \\
\hline & & Risco & Baixo risco & & & \\
\hline \multirow[t]{2}{*}{ Magreza } & Observados & 50 & 2 & 52 & \multirow{8}{*}{0,4099} & \multirow{8}{*}{1,78} \\
\hline & Esperados & 49,04 & 2,96 & 52,00 & & \\
\hline \multirow[t]{2}{*}{ Eutrofia } & Observados & 37 & 4 & 41 & & \\
\hline & Esperados & 38,67 & 2,33 & 41,00 & & \\
\hline \multirow[t]{2}{*}{ Sobrepeso } & Observados & 29 & 1 & 30 & & \\
\hline & Esperados & 28,29 & 1,71 & 30,00 & & \\
\hline \multirow[t]{2}{*}{ Total } & Observados & 116 & 7 & 123 & & \\
\hline & Esperados & 116,00 & 7,00 & 123,00 & & \\
\hline
\end{tabular}

RV: razão de verossimilhança. Fonte: Dados da pesquisa.

Nas Tabelas 3 e 4, são apresentados os achados de IMC analisados segundo o risco de desenvolvimento da doença renal crônica, comparados por testes de correlação e verossimilhança. Os valores esperados mostram como os dados deveriam ser distribuídos assumindo a hipótese nula. Os valores observados demonstram a distribuição dos dados coletados. Valores positivos de X 2 mostram que houveram mais casos observados do que o esperado, já valores negativos que menos casos foram observados em relação ao que era esperado. $\mathrm{O}$ valor de $\mathrm{X}^{2}$ de cada célula é somado para obter o $\mathrm{X}^{2}$ da tabela, que posteriormente é verificado na tabela de qui-quadrado (i.e., padrão) para achar o valor de p. Os dados foram considerados significativos quando $\mathrm{p}$ foi menor que 0,05 .

Após a aplicação do questionário SCORED, que avalia o risco de desenvolver Doença Renal Crônica associado às aferições antropométricas, que avalia o estado nutricional, a análise estatística qui-quadrado foi feita para testar a hipótese de associação entre valores supracitados. Ao analisar as Tabelas, a estatística de associação entre o estado nutricional de adultos e idosos com o risco do desenvolvimento da Doença Renal Crônica, o mesmo não apresentou associações significativas, sendo o valor de significância $\mathrm{P}<0,5$. Os dados na Tabela 3 e 4 se assemelham diretamente, contendo classificação de IMC (subdividido nos estágios da vida: adultos e idosos), separados conforme o risco ou baixo risco de desenvolver DRC, divididos entre os resultados observados após a pesquisa e os dados esperados antes do estudo ser realizado.

Tabela 5: Nível de adequação dos parâmetros bioquímicos avaliado em pacientes hospitalizados com risco de Doença Renal Crônica. Fortaleza, 2021. (n: 260).

\begin{tabular}{|c|c|c|c|c|c|c|}
\hline \multirow{2}{*}{ Parâmetros: } & \multicolumn{2}{|c|}{ VRA: } & \multicolumn{2}{|c|}{ VRAR: } & \multicolumn{2}{|c|}{ VRADR: } \\
\hline & $\mathbf{N}$ & $\%$ & $\mathbf{N}$ & $\%$ & $\mathbf{N}$ & $\%$ \\
\hline Magnésio: homem & 70 & 26,9 & 58 & 22,3 & 23 & 8,9 \\
\hline Magnésio: mulher & 53 & 20,4 & 11 & 4,2 & 45 & 17,3 \\
\hline Potássio: & 137 & 52,7 & 39 & 15 & 84 & 32,3 \\
\hline Fósforo: & 113 & 43,5 & 32 & 12,3 & 115 & 44,2 \\
\hline Cálcio: & 111 & 42,7 & 117 & 45 & 32 & 12,3 \\
\hline Sódio: & 101 & 38,8 & 60 & 23,1 & 99 & 38,1 \\
\hline
\end{tabular}

VRA: Valores de Recomendação Adequados. VRAR: Valores de Recomendações Abaixo do Recomendado. VRADR: Valores de Recomendação Acima do Recomendado. Fonte: Dados da pesquisa. 
Na Tabela 5, pode-se observar quanto nível de adequação dos parâmetros bioquímicos que 26,9\% (70) dos homens apresentam valores de recomendação adequados quanto aos valores de magnésio, já o percentual de adequação frente aos valores de referência para as mulheres é de 20,4 (53). Os valores coletados quanto aos níveis de potássio, metade da amostra, 52,7\% (137) apresentam valores dentro da recomendação. Quanto ao fósforo, 44,2\% (115) apresentam valores de recomendação acima dos valores de referência. Em relação aos valores coletados de cálcio a partir do acesso aos valores disponíveis nos prontuários, observou-se que 42,7\% (111) da população avaliada apresentam valores de recomendação adequados. Quanto aos valores de sódio, observa-se que 38,8\% (101) valores de recomendação adequados.

Em um estudo realizado em 2014 no estado de Santa Catarina foi observado que em todos os grupos de pacientes analisados haviam altas concentrações de fosfato, já que na DRC, a diminuição progressiva de néfrons funcionais induz a retenção de fosfato, que ao atingir a fase terminal manifesta-se com a hiperfosfatemia seja, o fósforo acima de 2,1 mmol/L, é uma característica comum da DRC (GIACHINI et al., 2017). Fazendo um comparativo, 44,23\% dos voluntários também possuem níveis de fósforo acima do recomendado, sendo essa alteração característica de portadores de DRC.

Tabela 6: Biomarcadores de função renal (Creatinina e Ureia), coletados pelos prontuários hospitalares dos pacientes hospitalizados. (n: 260). Fortaleza, 2021.

\begin{tabular}{|c|c|c|c|c|c|}
\hline \multirow{2}{*}{ Biomarcador: } & \multirow{2}{*}{ Sexo: } & \multicolumn{2}{|c|}{ Adequado: } & \multicolumn{2}{|c|}{ Inadequado } \\
\hline & & $\mathbf{N}$ & $\%$ & $\mathbf{N}$ & $\%$ \\
\hline \multirow[t]{2}{*}{ Creatinina: } & Masculino: & 95 & 36,5 & 56 & 21,5 \\
\hline & Feminino: & 67 & 25,8 & 42 & 16,2 \\
\hline Ureia: & & 97 & 37,3 & 163 & 62,7 \\
\hline
\end{tabular}

Fonte: Dados da pesquisa.

A partir dos dados coletados quantos aos biomarcadores de função renal: Creatinina e Ureia, descritos na Tabela 6, observou-se que 36,5\% (95) do sexo masculino estavam com valores dentro do recomendado de Creatinina, e já 25,8\% (67) do sexo feminino apresentavam valores ideais quanto a esse parâmetro. Em relação aos níveis de ureia coletados, observou-se que $62,7 \%$ (163) em ambos os sexos apresentam valores inadequados quanto a esse biomarcador.

A ureia é o principal metabólito nitrogenado derivado da degradação de proteínas pelo organismo, sendo $90 \%$ excretados pelos rins e correspondendo a aproximadamente 75\% do nitrogênio não-protéico secretado. O restante da ureia é eliminado pelo trato gastrointestinal e pela pele. A principal utilidade clínica da ureia parece estar na determinação em conjunto com a creatinina. A razão ureia sérica/creatinina sérica pode indicar estados patológicos diferentes. Valores abaixo do recomendado podem ser encontrados em patologias como a necrose tubular aguda, baixa ingestão de proteínas, condições de privação alimentar ou redução da síntese de ureia por insuficiência hepática (Da Silva et al., 2020).

A análise dessa razão elevada pode ser feita de forma dicotomizada com a creatinina dentro do valor de referência, indicando processos que levam a redução do fluxo sanguíneo renal, aumento na ingestão protéica, ou sangramento gastrintestinal; e com a creatinina acima do valor normal, denotando processos obstrutivos pós-renais, como tumores ou estenose de vias urinárias. Outra utilidade da ureia está na sua dosagem urinária, que pode fornecer informação crucial no campo da nutrição e tem sido utilizada em pacientes internados para monitoramento de dietas especiais (Da Silva et al., 2020). 
Tabela 7: Biomarcador de função renal (Taxa Filtrante Glomerular), coletados pelos prontuários hospitalares dos pacientes hospitalizados. Fortaleza, 2021, (n: 260).

\begin{tabular}{|c|c|c|c|}
\hline Biomarcador: TFG & Estágios: & $\mathbf{N}$ & $\%$ \\
\hline \multirow{6}{*}{$\begin{array}{l}\text { Taxa de Filtração } \\
\text { Glomerular: }\end{array}$} & Estágio 1 - (> $\left.90 \mathrm{ml} / \mathrm{min} / 1.73 \mathrm{M}^{2}\right)$ & 92 & 35,4 \\
\hline & Estágio 2 - $\left(60-89 \mathrm{ml} / \mathrm{min} / 1.73 \mathrm{M}^{2}\right)$ & 111 & 42,7 \\
\hline & Estágio 3A - (45-59 ml/min/1.73 M²) & 23 & 8,8 \\
\hline & Estágio 3-B - (30-44 ml/min/1.73 $\left.\mathrm{M}^{2}\right)$ & 24 & 9,3 \\
\hline & Estágio 4 - (15-29 ml/min/1.73 M²) & 10 & 3,8 \\
\hline & Estágio 5 - (<15 ml/min/1.73 M²) & 0 & 0 \\
\hline
\end{tabular}

Fonte: Dados da pesquisa.

$\mathrm{Na}$ Tabela 7, observou-se quanto ao biomarcador de função renal (Taxa Filtrante Glomerular) que 42,7\% (111) da população encontrava-se no Estágio $2\left(60-89 \mathrm{ml} / \mathrm{min} / 1.73 \mathrm{M}^{2}\right)$, sendo assim esse biomarcador é de suma importância para analisar os níveis de funcionalidade dos rins em relação a excreção, filtração etc. Assim, recomenda-se a realização de exames complementares como: hemograma, saturação de transferrina, ferritina sérica, no que diz respeito à micronutrientes em nível sérico, para pacientes com risco e diagnóstico de DRC (Peixoto et al., 2019).

O paciente com anormalidades nas funções renais apresenta alterações sistêmicas como: anemia, deficiência vitamina D, edema, restrição hídrica, hipercalemia, hiperfosfatemia, proteinúria, uremia, entre outros patologias associadas, sendo necessário monitorar os parâmetros bioquímicos destes pacientes para minimizar complicações e piores prognóstico clínicos. (Cuppari; 2019) 
Tabela 8: Questionário de frequência alimentar, com alimentos de fonte proteica, aplicados nos pacientes hospitalizados (n: 260). Fortaleza, 2021.

\begin{tabular}{|c|c|c|c|c|c|c|c|c|}
\hline \multirow{3}{*}{ Grupos Alimentar: } & \multicolumn{8}{|c|}{ Frequência: } \\
\hline & \multicolumn{2}{|c|}{0} & \multicolumn{2}{|c|}{$1-3$} & \multicolumn{2}{|c|}{$3-5$} & \multicolumn{2}{|c|}{$5-7$} \\
\hline & $\mathrm{N}$ & $\%$ & $\mathrm{~N}$ & $\%$ & $\mathrm{~N}$ & $\%$ & $\mathrm{~N}$ & $\%$ \\
\hline Alimentos industrializados & 58 & 22,3 & 102 & 39,2 & 54 & 20,7 & 46 & 17,6 \\
\hline Bebidas e bebidas alcoólicas: & 33 & 12,6 & 65 & 25 & 54 & 20,7 & 108 & 41,5 \\
\hline Enlatados ou em conserva: & 66 & 25,3 & 113 & 43,4 & 51 & 19,6 & 30 & 11,5 \\
\hline Temperos industrializados: & 37 & 14,2 & 33 & 12,7 & 34 & 13,1 & 156 & 60 \\
\hline Comidas Prontas/fast food: & 67 & 25,8 & 109 & 41,9 & 63 & 24,2 & 21 & 8,1 \\
\hline Proteína Vegetal: & 3 & 1,1 & 12 & 4,6 & 20 & 7,7 & 225 & 86,5 \\
\hline Carne vermelha: & 4 & 1,5 & 23 & 8,9 & 47 & 18,1 & 186 & 71,5 \\
\hline Carnes brancas: & 2 & 0,9 & 31 & 11,9 & 54 & 20,7 & 173 & 66,5 \\
\hline Lacticínios: & 17 & 6,5 & 28 & 10,8 & 22 & 8,5 & 193 & 74,2 \\
\hline Oleaginosas: & 76 & 29,2 & 116 & 44,6 & 48 & 18,5 & 20 & 7,6 \\
\hline Embutidos: & 49 & 18,8 & 85 & 32,7 & 57 & 21,9 & 69 & 26,6 \\
\hline Ovo: & 1 & 0,9 & 35 & 13,5 & 46 & 17,3 & 179 & 68,8 \\
\hline
\end{tabular}

Fonte: Dados da pesquisa.

Na Tabela 8, observa-se a partir da aplicação do questionário de frequência alimentar, quanto ao consumo de proteínas de origem vegetal 71,5\% (186) relataram consumir de 5 - 7 dias da semana, quanto ao grupo de lacticínios: observase que 74,2\% (193) consomem alimentos como: leite, queijo, requeijão, nata, coalhada ou iogurte 5 - 7 dias da semana.

No estudo realizado por (Paiva et al., 2020), a respeito da Avaliação do consumo alimentar e das condições socioeconômicas de pacientes sob terapia de hemodiálise, o consumo alimentar foi avaliado a partir do questionário de frequência alimentar para pacientes em diálise (Riella \& Martins, 2013). Quanto ao consumo de cereais e leguminosas, 100\% dos participantes do estudo consomem arroz. O arroz é um alimento produzido e consumido em todo o território brasileiro, e junto ao feijão, é um dos alimentos básicos da alimentação brasileira. Quanto ao consumo de laticínios e ovos, o maior percentual de consumo nesse grupo é de ovos com 53,20\%, seguido por 31,25\% quanto a ingestão de leite e subsequente $25 \%$ dos participantes relataram consumir queijo. Quanto ao consumo de carnes neste estudo, o consumo de aves é de 59,40\%, $37,50 \%$ consomem carne vermelha, $34,40 \%$ consomem peixes e $21,90 \%$ consomem outros tipos.

Segundo Pereira et al., 2020, o consumo de potássio e de fósforo merece atenção na prevenção e durante o tratamento da DRC, esses micronutrientes podem ser encontrados em laticínios e carnes, que são alimentos de origem animal e nos alimentos de origem vegetal, como frutas, nozes e legumes. Na fase mais avançada da DRC, a hipercalemia (concentração de potássio no sangue acima do valor de referência, 3,5-5,5 mEq/ L) e a hiperfosfatemia (concentração de fósforo no sangue acima do valor de referência, 2,5 e 4,5 mg/dL) prevalecem. Com a restrição da excreção renal, alguns fatores favorecem para o ganho do potássio e fósforo séricos, sendo eles, a acidose metabólica, constipação intestinal, diabetes mellitus, assim como a ingestão de alimentos com alto teor de potássio. 
Logo, quanto ao consumo de carnes vermelhas podemos observar que 71,5\% (186) relataram consumir de 5 - 7 dias da semana. Em relação ao grupo das oleaginosas: amendoim, castanhas e amêndoas, pode-se observar que o maior percentual de consumo nesse grupo foi de 44,6\% (116) essa parcela da amostra relatou consumir esse grupo de alimentos de 1 - 3 dias da semana. Em relação ao consumo de carnes brancas: aves, peixes e crustáceos, podemos observar no presente estudo que $66,5 \%$ (173) relataram consumir de 5 - 7 dias da semana. No que diz respeito ao consumo de ovos observa-se que 68,8\% (179) relataram consumir de 5 - 7 dias da semana. O ovo é considerado uma fonte de proteína barata, de fácil acesso e de alta qualidade, às populações de países de baixa e média renda tem os ovos como uma boa fonte de proteína acessível, que pode explicar o maior consumo e preferência, este alimento é fonte do aminoácido leucina, sendo fundamental para a síntese de proteínas musculares (Tallman et al., 2018).

Segundo Banerjee, a dieta é um fator essencial e determinante podendo trazer vários benefícios para os pacientes com risco de desenvolver DRC, visando assim a manutenção do equilíbrio ácido-básico (Banerjee et al., 2016). Uma alimentação rica em proteína animal tende a gerar um aumento na produção de ácido, já a alimentação rica em vegetais e frutas geram bicarbonato e reduzem a carga líquida de ácidos na dieta. No estudo de Pereira, a restrição do aporte protéico tem sido bastante utilizada na prescrição dietética dos pacientes com DRC. Estudos mostram que o consumo elevado de proteína aumenta o fluxo plasmático dos rins, a taxa de filtração glomerular, a pressão intraglomerular e influência na hemodinâmica renal, e a sua diminuição pode auxiliar no controle da DRC, favorecendo assim a melhoria nos níveis de albuminúria e no controle da uremia (Pereira et al., 2020).

Na Tabela 8, em relação ao consumo de alimentos de alto teor de sódio como alimentos industrializados 39,2\% (102) relatou consumir de 1 - 3 dias da semana esse grupo de alimentos como salgadinhos de pacote, macarrão instantânea ou biscoitos. Em relação ao consumo de bebidas como refrigerantes, sucos artificiais, destilados e fermentados, observa-se que 41,5\% (108) relataram consumir as bebidas citadas de 5 - 7 dias da semana. Quanto aos alimentos enlatados ou em conserva, pode-se observar que 43,3\% (113) da população estudada, relatou consumir de 1 - 3 dias da semana esse grupo de alimentos como azeitonas, milho, palmito, carne de lata ou tomate seco. Em relação ao consumo de alimentos embutidos como presunto, salame, mortadela, nuggets, salsicha e linguiça, podemos observar na tabela (Tabela 6) que 32,7\% (85) relataram consumir de 1 - 3 dias da semana esse grupo de alimentos.

Logo, quanto aos temperos industrializados, pode-se notar que 60\% (156) relataram utilizar durante o preparo de suas refeições de 5 - 7 dias da semana. Quanto ao consumo de comidas prontas/ fast food como: pastel, hot dog, pizza ou hambúrguer, 41,9\% (109) da amostra estudada, relatou consumir de 1 - 3 dias da semana esse grupo de alimentos. Assim, a restrição de sódio é recomendada com objetivo reduzir o ganho de peso interdialítico, sendo recomendado de 5 a $6 \mathrm{~g}$ de sal (cloreto de sódio) por dia, ou seja, 2.000 a $2.300 \mathrm{mg} / \mathrm{dia}$, metade da quantidade que é ingerida pelos brasileiros, justificando a dificuldade dos pacientes a seguirem essa orientação, desta forma, visando a redução no consumo, os profissionais devem orientar seus pacientes a realizarem trocas de temperos industrializados por temperos naturais, ressaltando também que eles não devem consumir produtos em conservas, embutidos, enlatados, refrigerantes, sucos artificiais e temperos industrializados por terem um alto teor de sódio (Cuppari, 2019). 
Tabela 9: Nível de ingestão hídrica pelos pacientes hospitalizados (n: 260). Fortaleza,2021.

\begin{tabular}{l|c|c|}
\hline Nível de hidratação/ingestão hídrica & \multicolumn{2}{c}{$\mathbf{N}$} \\
\hline Ingestão satisfatória: $\geq 21$ & 28 & 10,7 \\
Ingestão moderado: $>1$ ou $<2$ l & 100 & 38,5 \\
Ingestão insuficiente: $<1$ l & 132 & 50,8 \\
\hline
\end{tabular}

Fonte: Dados da pesquisa.

Cerca de $60 \%$ do corpo humano é composto por água, cuja ingestão adequada é essencial para a estrutura e função dos tecidos, homeostase e regulação do metabolismo (Lucchesi et al., 2021). A Organização Mundial da Saúde (OMS) orienta o consumo para adultos saudáveis de em média dois litros por dia. Apesar da recomendação da OMS, a quantidade de água ideal deve ser específica para cada indivíduo, dependendo de suas atividades diárias, seu estado de saúde e a proporção do peso. Uma pessoa que faz exercícios físicos e simultaneamente perde água pela transpiração, necessita de maior hidratação quando comparada a uma pessoa que passa grande parte do dia em repouso. Uma maior hidratação também é recomendada para pessoas com queimaduras, com diarreia prolongada, febre, que consomem álcool, que fazem uso de diuréticos e diabéticos (Guimarães et al., 2021). Na Tabela 9, mais da metade da amostra estudada, 50,8\% (132) da amostra estudada relatou ingestão hídrica menor que 1 litro por dia.

O Panel on Dietetic Products, Nutrition, and Allergies (NDA) da European Food Safety Authority (EFSA) recomenda valores de referência para a quantidade de ingestão de água por dia, se baseando em valores de consumo de água total por diversos países europeus, valores necessários de osmolaridade urinária e da água por energia consumida. Esses valores de Ingestão Adequada (AI), são aplicados para adultos em relação a atividade física e condições climáticas moderadas, sendo considerado valores como: ideal 2,0L/dia para mulheres e 2,5L/dia para homens (EFSA, 2010).

\section{Conclusão}

Por conseguinte, os resultados da pesquisa refletem que não há correlação de forma direta entre o estado nutricional do paciente hospitalizado com o risco desenvolvimento da Doença Renal Crônica (DRC), pois o valor estatístico de correlação foi contrário ao esperado, sendo necessário novos estudos, preferencialmente mais aprofundados com o objetivo de correlacionar os parâmetros avaliados com uma nova perspectiva e se possível, com novas ferramentas. Segundo as informações dispostas supracitadas pode-se inferir que a maioria dos avaliados possui o risco de desenvolver DRC, conforme constatou-se no questionário SCORED. Em relação aos biomarcadores, os valores de ureia apresentaram-se potencialmente inadequados, uma quantidade expressiva estava com TFG em estágio 2.

A monitorização e reavaliação frequente dos pacientes por parte dos profissionais de nutrição devem ocorrer assiduamente, para estabelecer pontos críticos e com isso implantar uma terapia nutricional eficaz. O acompanhamento nutricional visa promover uma melhor qualidade de vida, minimizando os riscos nutricionais em âmbito hospitalar. Atentar-se na prescrição dietética adequada, mediante as carências energéticas e nutricionais, visando a manutenção e recuperação do estado nutricional do paciente, controlando principalmente a ingestão de líquidos, sódio, potássio e proteínas A intervenção dietética deve ser individual e adaptada de acordo ao estágio da patologia, sabe-se que na maioria das vezes é difícil a aceitação e a adesão dessa dieta pelos pacientes, por isso salienta-se a importância da orientação dos profissionais de saúde junto aos familiares.

É necessário que sejam desenvolvidos mais estudos científicos acerca do desenvolvimento de Doenças Renais Crônicas, para assim colaborar com a comunidade científica. 


\section{Referências}

Aquino, R. C. \& Philippi, S. T. (2011). Identificação de fatores de risco de desnutrição em pacientes internados. Revista da Associação Médica Brasileira, $57(6), 637-643$

Banerjee, T., Liu, Y., \& Crews, D. C. (2016). Dietary patterns and CKD progression. Blood purification, 41(1-3), 117-122.

Bastos, M. G., \& Kirsztajn, G. M. (2011). Doença renal crônica: importância do diagnóstico precoce, encaminhamento imediato e abordagem interdisciplinar estruturada para melhora do desfecho em pacientes ainda não submetidos à diálise. Brazilian Journal of Nephrology, 33, 93-108.

Bousquet-Santos, K., Costa, L. D. G. D., \& Andrade, J. M. D. L. (2019). Estado nutricional de portadores de doença renal crônica em hemodiálise no Sistema Único de Saúde. Ciência \& Saúde Coletiva, 24, 1189-1199.

Brasil. (2014a) Ministério da Saúde. Secretaria de Atenção à Saúde. Departamento de Atenção Especializada e Temática. Diretrizes Clínicas para o Cuidado ao paciente com Doença Renal Crônica - DRC no Sistema Único de Saúde/ Ministério da Saúde. Secretaria de atenção à saúde. departamento de atenção especializada e temática. 37.

Brasil. (2014b) Ministério da Saúde. Secretaria de Atenção à Saúde. Departamento de Atenção Básica. Guia alimentar para a população brasileira / Ministério da Saúde, Secretaria de Atenção à Saúde, Departamento de Atenção Básica.

Campelo, D. E., Vasconcelos, R. C. \& Cabral, M. A. (2007). Terapia Nutricional. Em: Condutas em Clínica Médica. Filgueira N. A. et al. (4a Ed). Rio de Janeiro: Guanabara Koogan, 759-771.

Cecconelo, L., Winkelmann, E. R., Krug, R. R., Moreira, P. R. (2019) Perfil Clinico- Epidemiologico dos doentes renais crônicos em tratamento hemodialitico: Um estudo da região noroeste do estado do Rio Grande do Sul. Temas em Sáude. 19(3).

Cozzolino, S. M., \& Cominetti, C. (2019). Bases bioquímicas e fisiologica da nutrição: nas diferentes fases da vida, na saúde e na doença. Manole. Cuppari, L., (2019). Guia de Nutrição: nutrição clínica no adulto. (4a Ed.). Manole.

Da Silva, J. A.; \& Santos, L. S. C. (2020). Monitorização da função renal de pacientes internados em Unidades de Terapia Intensiva em uso de antimicrobianos/Renal function monitoring of patients admitted to Intensive Care Units using antimicrobials. Arquivos Médicos dos Hospitais e da Faculdade de Ciências Médicas da Santa Casa de São Paulo. 65(1), 1-9.

EFSA Panel on Dietetic Products Nutrition and Allergus (NDA) (2010) Scientific Opinion on Dietary reference values for water. EFSA jornal 8, 1459.

ESPEN guideline on clinical nutrition in hospitalized patients with acute or chronic kidney disease (2021). Clinical Nutrition, 40(4), 1644-1668.

Fiaccadori, E., Sabatino, A., Barazzoni, R., Carrero, J. J., Cupisti, A., De Waele, E., \& Cuerda, C. (2021). ESPEN guideline on clinical nutrition in hospitalized patients with acute or chronic kidney disease. Clinical Nutrition, 40(4), 1644-1668.

Giachini, E., Zanesco, C., Souza, S. S. d., Ceratto, P. C., \& Silva, D. T. d. R. e. (2017). Perfil De Pacientes Hemodialíticos Relacionados a Parâmetros Bioquímicos e Hematológicos No Oeste Catarinense. Colloquium Vitae, 9(2).

Guimarães, B. P., Maciel, A. R., Barbosa, A. P., Viziolli, B. N. P., Vitta, C. E., \& Chaud, D. M. A. (2021). O consumo de água em idosos: uma revisão. Vita et Sanitas, 15(2), 53-69.

Krause, M. V., Mahan, L. K., \& Escott-Stump, S. (2018). Krause. Alimentos, Nutrição e Dietoterapia. (14a Ed.) GEN Guanabara Koogan.

Lucchesi, I., Fisberg, R. M., Sales, C. H., A qualidade da dieta está associada com a ingestão de água em residentes de São Paulo, Brasil. (2021). Ciência \& Saúde Coletiva, 26, 3875-3883.

Malta, D. C., Machado, Í. E., Pereira, C. A., Figueiredo, A. W., Aguiar, L. K. D., Almeida, W. D. S. D., \& Szwarcwald, C. L. (2019). Avaliação da função renal na população adulta brasileira, segundo critérios laboratoriais da Pesquisa Nacional de Saúde. Revista Brasileira de Epidemiologia, 22.

Paiva, A. A. et al. (2020). Avaliação do consumo alimentar e das condições socioeconômicas de pacientes sob terapia de hemodiálise no sertão paraibano. Research, Society and Development, 9(7), e747974855-e747974855.

Peixoto, A. B. S., Jaime, K. P. S. S., Neto, J. R. M., Costa, S. H. N., Penna, K. G. B. D. \& Gomes, C. M. (2019). Avaliação De Variáveis Analíticas Na Doença Renal Crônica Em Pacientes Atendidos Em Laboratórios De Goiânia - Goiás. Revista Brasileira Militar de Ciências, 5(12).

Pereira, R. A. et al. (2020). Diet in Chronic Kidney Disease: an integrated approach to nutritional therapy. Revista da Associação Médica Brasileira, 66, 59s67.

Riella M. C., \& Martins C. (2013). Nutrição e o Rim. (2a ed). Guanabara.

Rosa, A. C. S., Roque, J. R., \& Gonçalves, D. R. (2020). Estado Nutricional de Idosos Residentes em Instituição Geriátrica e a Relação com o Consumo Alimentar. Revista Contexto \& Saúde, 20(41), 25-35.

Santos, A. C. B. D., et al. (2013). Associação entre qualidade de vida e estado nutricional em pacientes renais crônicos em hemodiálise. Brazilian Journal of Nephrology, 35, 279-288.

Santos, P. R., \& Monte, V. L. (2016). Aplicação do questionário SCORED para rastreamento da doença renal crônica entre pacientes hipertensos e diabéticos. Cadernos Saúde Coletiva, 24, 428-434. 
Research, Society and Development, v. 10, n. 16, e287101623705, 2021

(CC BY 4.0) | ISSN 2525-3409 | DOI: http://dx.doi.org/10.33448/rsd-v10i16.23705

Sarmento, L. R., Fernandes, P. F. C. B. C., Pontes, M. X., Correia, D. B. S., Chaves, V. C. B., Carvalho, C. F. D. A., \& Moliterno, L. A. A. (2018). Prevalência das causas primárias de doença renal crônica terminal (DRCT) validadas clinicamente em uma capital do Nordeste brasileiro. Brazilian Journal of Nephrology, 40, 130-135.

Silva, D. J. S., Da Silva G., Marques V. A., Ferraz, J. R S. (2019). Perfil Nutricional de Idosos internados em um hospital público na cidade de Caxias-MA: Um relato de experiência. Research, Society and Development, 8(4), e3984931

Tallman, D. A., Sahathevan, S., Karupaiah, T., \& Khosla, P. (2018). Egg Intake in Chronic Kidney Disease. Nutrients, 10(12). (1945).

Thomé, F. S., Sesso, R. C., Lopes, A.A., Lugon, J. R., \& Martins, C. T. (2019). Brazilian chronic dialysis survey 2017. J Bras Nefrol. $41(2)$ :208-14.

Trivedi, H., Pang, M., Campbell, A., \& Saab, P. (2012). Slowing the progression of chronic renal failure: economic benefits and patients' perspectives. Am J Kidney Dis. ;39(4):721-9. http://dx.doi.org/10.1053/ ajkd.2002.31990.

Trivedi, H. (2010). Cost implications of caring for chronic kidney disease: are interventions cost-effective? Advances in chronic kidney disease, 17(3), 265270.

Vasconcelos, M. I. L., Fernandes, H. M. A., da Silva Barbosa, E., de Oliveira Grangeiro, R. F., de Sena, D. B. G., Lopes, V. C. B., \& Firmino, L. A. R. G. (2021). Nutrição e doença renal crônica (DRC): Apresentação das novas recomendações e padrões alimentares conforme as últimas evidências científicas. Research, Society and Development, 10(6), e28610615891-e28610615891.

Victor S, Barbosa P, Maria A, Barros E De, \& Martins L. (2015). Estimativa da taxa de filtração glomerular na prática clínica: revisão de literatura. RENOME 31-2. http://www.renome.unimontes.br/index.php/renome/article/view/19. 